\title{
Valores de Condición Física relacionada con la Salud en adolescentes de 14 a 17 años; relación con el estado de peso \\ Values of Health-related Physical Fitness in adolescents from 14 to 17 years of age; relationship with the weight status \\ Laura Nieto López, Eliseo Garcia Cantó, Andrés Rosa Guillamón \\ Universidad de Murcia (España)
}

\begin{abstract}
Resumen. El objetivo de este estudio fue proporcionar valores percentiles de la batería COFISA según el género y, su relación con el estado de peso. Es un estudio descriptivo con 1037 adolescentes (508 varones y 529 mujeres; 15,52 $\pm 1,11$ años). La prueba MANOVA reflejó diferencias a favor de los varones a excepción de la flexibilidad y el estado de peso en normopeso, donde las mujeres hallaron un mejor rendimiento físico. Con el avance de la edad se observa un incremento progresivo del nivel de condición física global. En la relación entre el estado de peso, coordinación y condición física según el sexo, los varones en normopeso mostraron mayores promedios que sus pares en sobrepeso y obesidad. Cabe destacar que el grupo en obesidad mostró valores más elevados en coordinación. Por su parte, las mujeres en normopeso obtuvieron registros superiores en fuerza manual y coordinación respecto al grupo en sobrepeso y obesidad, siendo el grupo en sobrepeso quien mostró registros superiores en abdominales, resistencia cardiovascular, flexibilidad, condición física global y coordinación respecto al grupo con normopeso; y en flexibilidad y coordinación en relación al grupo con obesidad, siendo el grupo con obesidad quien mostró valores más elevados en fuerza abdominal. En conclusión, este estudio proporciona percentiles actualizados que permiten evaluar la condición física relacionada con salud en adolescentes con el fin de identificar a aquellos en riesgo de enfermedades crónicas importantes, así como evaluar los efectos de intervenciones alternativas en este grupo etario.
\end{abstract}

Palabras clave. COFISA, adolescentes, condición física, estado de peso.

\begin{abstract}
The objective of this study was to provide gender-specific percentile values of the COFISA test, and their relation to weight status. This is a descriptive study with 1,037 adolescents ( 508 boys and 529 girls, $15.52 \pm 1.11$ years old). The MANOVA test showed differences in favor of boys, with the exception of flexibility and weight status in normal weight, where girls were found with a better physical performance. With increased age, a progressive rise in the level of overall fitness was observed. In the relationship between weight status, coordination, and physical fitness by gender, normal-weight boys showed higher averages than their overweight and obese peers. It should be noted that the obesity group showed higher values in coordination. Regarding girls, the normal-weight ones obtained higher scores in manual strength and coordination than the overweight and obese group, with the overweight group showing higher scores in abdominal strength, cardiovascular endurance, flexibility, overall physical condition, and coordination compared to the group with normal weight; and in flexibility and coordination compared to the group with obesity. The obesity group showed higher scores in abdominal strength. In conclusion, this study provides updated percentiles that allow the evaluation of health-related physical fitness in adolescents so to identify those at risk of major chronic diseases, as well as to evaluate the effects of alternative interventions in this age group.
\end{abstract}

Key Words. COFISA, teenagers; Physical fitness; Body composition.

\section{Introducción}

La adolescencia se caracteriza por importantes cambios psicológicos y fisiológicos que influyen sobre el comportamiento y el estilo de vida, tanto a medio como a largo plazo (Ortega, Ruiz, \& Castillo, 2013). Estos cambios que se experimentan durante el crecimiento se han asociado tanto al incremento de la masa corporal como al ejercicio físico desempeñado (Marrodán-Serrano et al., 2009; Lopes, Malina, GomezCampos, Cossio-Bolaños, de Arruda, \& Hobold, 2018). Durante este periodo, se ha descrito que la realización de actividad física regular favorece la menor acumulación de masa grasa, al tiempo que colabora con el aumento de la cantidad de tejido libre de grasa y del capital óseo, contribuyendo por tanto a que los escolares crezcan de una forma más saludable (Casajús et al., 2006). La capacidad que tiene una persona para realizar actividad física y/o ejercicio, la cual constituye una medida integrada de todas las funciones y estructuras que intervienen en su realización se denomina condición física (CF) (Ruiz et al. 2009). Los principales indicadores del

Fecha recepción: 31-12-18. Fecha de aceptación: 15-08-19 Eliseo Garcia Canto

eliseo.garcia@um.es estado de salud relacionado con la CF pueden ser la composición corporal, la capacidad motora, la capacidad aeróbica y la fuerza músculo-esquelética (Rosa Guillamón, 2017).

La evidencia científica refleja que estos indicadores han sufrido un alarmante descenso en sus niveles a excepción de la composición corporal (Ortega et al., 2008), siendo la obesidad el trastorno nutricional más frecuente en la adolescencia; asociado a numerosas comorbilidades tales como la hiperinsulinemia y la resistencia a la insulina; ambas son el eje central del desarrollo posterior de estados de intolerancia a la glucosa, diabetes tipo 2 y/o síndrome metabólico (Yeste \& Carrascosa, 2011; Palomino-Devia, Otero-Saborido, \& González-Jurado, 2016). Los métodos de medida de la composición corporal han mostrado las consecuencias en la salud del exceso de grasa, en especial de la grasa visceral, así como la influencia de este tejido en el perfil metabólico del obeso (Mundín, Lafita, Petrina, \& Argüelles, 2009). A su vez, la prevalencia de estudiantes con problemas en su desarrollo motor es cada vez mayor, asociándose a parámetros de salud mental como una menor autoestima, un peor autoconcepto, una baja percepción de competencia atlética, sufrir de ansiedad social y acoso escolar (Rosa-Guillamón, García-Cantó, \& Carrillo-López, 2018).

Por tanto, la identificación temprana de adolescentes con 
bajos niveles de CF puede permitir implementar intervenciones para promover comportamientos saludables a fin de prevenir el riesgo cardiometabólico en la edad adulta (Gualteros, Torres, Umbarila-Espinosa, Rodríguez-Valero, \& RamírezVélez, 2015) y conseguir de esta manera altos niveles de CF, los cuales están relacionados con mayor salud cardiovascular en adolescentes (Secchi, García, España-Romero, \& CastroPiñero, 2014). La evidencia científica sugiere que las intervenciones de monitoreo de la $\mathrm{CF}$ y el crecimiento físico deben estar diseñadas para alcanzar metas saludables e incluir acciones de acuerdo con las características socioculturales y ambientales de los jóvenes (Guedes, Franzini, Júnior, \& Morales, 2017). Se hace necesario desde una perspectiva práctica, la selección mediante criterios objetivos de una batería de pruebas adecuadamente validadas que permitan la valoración óptima de las principales cualidades fisiológicas del sujeto y fáciles de realizar en el contexto escolar, así como en estudios epidemiológicos (Rosa-Guillamón, 2017).

En este sentido, uno de los protocolos desarrollados recientemente ha sido la Batería de test de condición física relacionada con la Salud (COFISA), la cual integra de manera conjunta las capacidades coordinativas y condicionales. Por otro lado, es destacable que la fiabilidad intra e interobservador y la validez de sus pruebas hacen que sea una batería adecuada para ser utilizada en investigaciones sobre mediciones del nivel de condición física saludable tanto en escolares de primaria (Yuste y Rodríguez, 2012) como en secundaria (Lazaro, 2011). Además, se trata de una batería que, con respecto a otras pruebas, es fácilmente adaptable al entorno escolar, ya que está compuesta por pruebas simples, poco costosas y de fácil realización (Barrancos, 2008).

En base a estos precedentes y, considerando que un nivel bajo de CF y, especialmente la obesidad repercute en la salud pública y está fuertemente relacionada con diversos desórdenes fisiológicos y psicosociales que configuran y condicionan el estilo de vida durante la adultez (Ruiz et al., 2011), el objetivo de este estudio fue proporcionar valores percentiles del test de valoración de las capacidades coordinativas y condicionales (COFISA) en adolescentes de 14 a 17 años según el sexo y el estado de peso corporal.

\section{Material y método}

\section{Participantes}

De una población total de 64015 sujetos, fueron seleccionados 1037 adolescentes de la Región de Murcia (véase tabla 1). 508 varones y 529 mujeres con edades comprendidas entre los 14 y los 17 años ( $\mathrm{M} \pm \mathrm{DE}=15,52 \pm 1,13$ años $)$ participaron en este estudio empírico descriptivo y transversal ex post facto. El muestreo fue de tipo aleatorio. Los criterios de exclusión se basaron en el diagnóstico clínico de los siguientes casos: (1) enfermedades que pudieran suponer un riesgo ante el esfuerzo físico intenso; (2) embarazo; y (3) uso de drogas. Los adolescentes y sus padres/tutores fueron informados de las características del estudio y firmaron un consentimiento informado.

\section{Variables $e$ instrumentos}

Evaluación de la condición relacionada con la salud La aptitud física de los participantes para ejecutar los

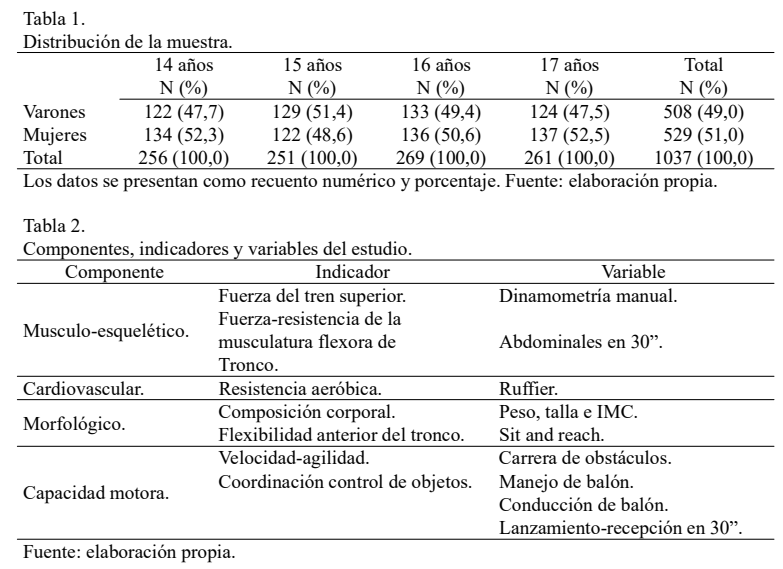

test de campo se evaluó mediante el Cuestionario de Aptitud para la Actividad Física (Thomas, Reading y Shepard, 1992). Se administraron las pruebas integradas en la batería Condición Física relacionada con la Salud (COFISA; López-Barrancos, 2008; véase tabla 2). La fiabilidad y validez de las pruebas demuestra su capacidad para realizar una valoración objetiva y completa de la condición física general en sujetos de entre 14 y 17 años de edad (Lázaro-Martínez, 2011). Además, tienen una relación directa con la salud según los postulados del American College of Sport Medicine (Amstrong, Whaley, Brubaker y Otto, 2005). Todos los test se administraron dos veces y se registró la mejor marca, excepto en la prueba de resistencia aeróbica que se administró una sola vez.

Los registros de las pruebas condicionales (dinamometría, Ruffier y sit and reach) fueron recodificados a unos valores ajustados por sexo y edad entre 0 y 10 . Con estos valores se calculó una media para obtener la puntuación de la variable condición física. De manera similar, se calculó una media de las variables que miden las capacidades coordinativas (carrera de obstáculos, manejo de balón, conducción de balón y lanzamiento-recepción) para obtener la variable de coordinación, con unos valores también entre 0 y 10.

\section{Procedimiento}

Antes del estudio se realizaron talleres de entrenamiento de dos exploradores colaboradores para la estandarización de las medidas. Se informó a los participantes que no modificasen sus hábitos de vida durante los días previos a la administración de las pruebas. Se realizó un calentamiento de 8 minutos basado en movilidad articular dinámica. Se permitió un intento de cada prueba. El estudio fue realizado durante el curso académico (2017/18) administrando las pruebas durante el mes de octubre. Además, este estudio se basó en las directrices éticas de la Declaración de Helsinki (revisión de 2013). La investigación pertenece a una tesis doctoral que se está desarrollando en el Departamento de Expresión Plástica, Musical y Dinámica de la Universidad de Murcia (Murcia, España).

\section{Análisis estadístico}

La distribución de las variables resulto normal tras aplicar la prueba de normalidad de Kolmogorov- Smirnov ( $p>$ $0,05)$; por consiguiente, se procedió a utilizar estadística 
paramétrica. El análisis descriptivo se realizó mediante el cálculo de estadísticos básicos: media, desviación estándar, percentiles, porcentajes y recuento numérico. Las diferencias entre grupos fueron estudiadas mediante un análisis multivariante de la varianza (MANOVA). En el análisis de las diferencias según el estado de peso, el valor de $p$ de los contrastes de hipótesis post-hoc se determinó mediante la corrección de Bonferroni para comparaciones múltiples. Se estableció un intervalo de confianza (IC) del 95\%. Los datos fueron analizados con el programa estadístico Statistical Package for the Social Sciences (v.23.0 de SPSS Inc., Chicago, IL, EE.UU.). El nivel de significación se fijó en el 5\% $(p \mathrm{~d} \gg 0,05)$.

\section{Resultados}

La prueba MANOVA mostró diferencias estadísticamente significativas a favor de los varones en un mejor rendimiento físico en los test de dinamometría manual, abdominales, Ruffier, carrera de obstáculos, conducción de balón y lanzamiento-recepción ( $p$ entre $<0,05 \mathrm{y}<0,001) \mathrm{y}$ talla, peso e IMC ( $p$ entre $<0,05 \mathrm{y}<0,001)$, obteniendo las mujeres un mejor rendimiento físico en sit and reach y mayor prevalencia de normopeso ( $p$ entre $<0,01 \mathrm{y}<0,001$ ). Respecto a la edad, los varones obtuvieron un mayor IMC a los 14 años $(p<0,001)$, a los 16 años, en coordinación $(p<0,05)$; y en los 17 años en condición física global $(p<0,001)$ mientras que las mujeres a los 15 años obtuvieron mayor IMC ( $p<$ $0,05)$ (véase tabla 3 ).

En las tablas 4 y 5 se muestran los valores referencia de las variables de la batería COFISA clasificados por sexo y edad expresados en percentiles del 10 al 90. En general, se observa una tendencia hacia el incremento en la condición física global (COFISA) en ambos sexos, aunque en alguna
Tabla 4 .

Valores percentiles de la batería Condición Física relacionada con la Salud (COFISA) en varones de 14-17 años.

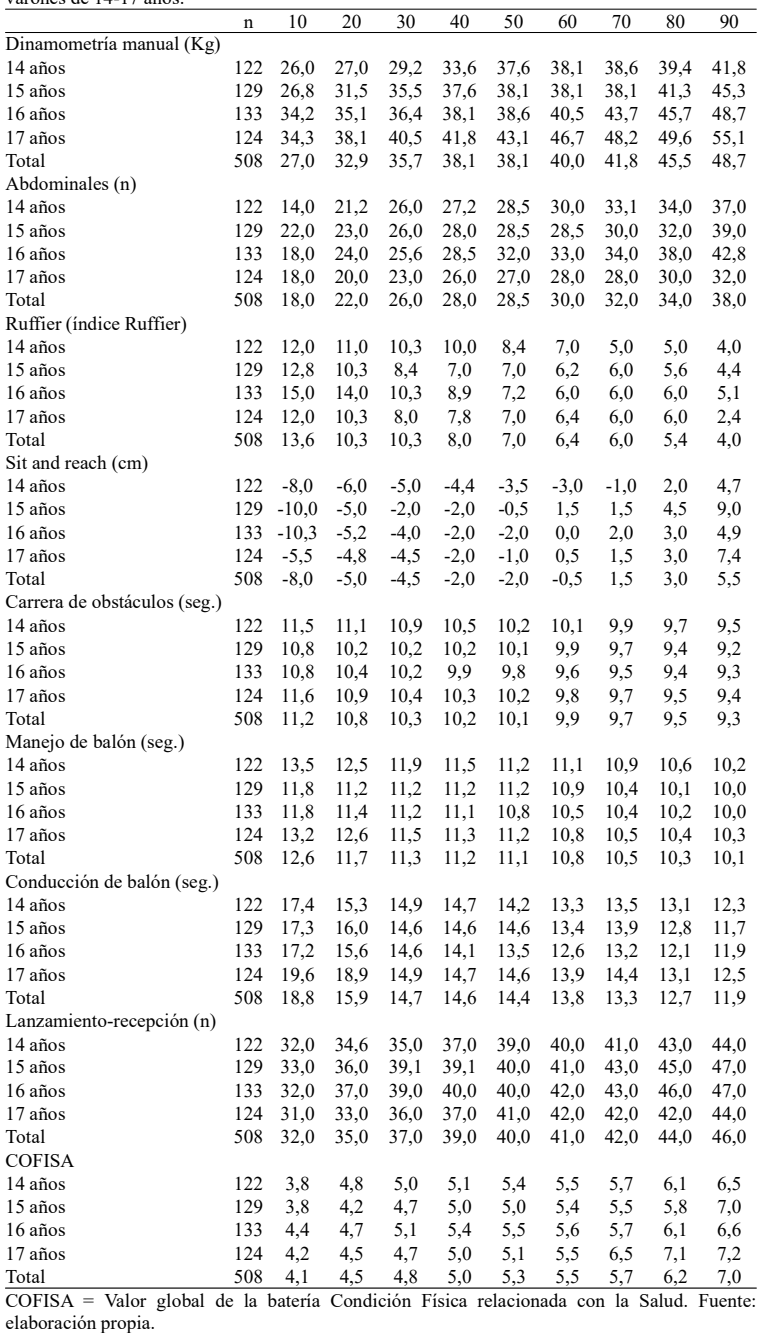

Tabla 3

\begin{tabular}{|c|c|c|c|c|c|}
\hline & & $\begin{array}{c}14 \text { años } \\
(n=256)\end{array}$ & $\begin{array}{c}15 \text { años } \\
(n=251)\end{array}$ & $\begin{array}{c}16 \text { años } \\
(n=269)\end{array}$ & $\begin{array}{c}17 \text { años } \\
(n=261)\end{array}$ \\
\hline \multicolumn{6}{|l|}{ Condición física } \\
\hline \multirow{2}{*}{ Dinamometría manual (kg) } & Varones & $35,0 \pm 6,7^{* * *}$ & $36,9 \pm 7,2^{* * *}$ & $40,6 \pm 6,6^{* * *}$ & $43,4 \pm 8,2 * * *$ \\
\hline & Mujeres & $25,8 \pm 5,1$ & $29,0 \pm 6,1$ & $28,5 \pm 6,1$ & $33,8 \pm 4,7$ \\
\hline \multirow{2}{*}{ Abdominales (n) } & Varones & $27,5 \pm 9,5^{* *}$ & $28,8 \pm 7,4^{* * *}$ & $29,8 \pm 9,7^{* * *}$ & $24,4 \pm 8,7^{* * *}$ \\
\hline & Mujeres & $23,3 \pm 9,7$ & $23,4 \pm 9,2$ & $25,4 \pm 8,0$ & $19,0 \pm 7,8$ \\
\hline \multirow{2}{*}{ Ruffier (indice de Ruffier) } & Varones & $8,3 \pm 3,4 * * *$ & $7,8 \pm 3,4^{*}$ & $8,97 \pm 4,31^{*}$ & $6,9 \pm 3,2^{* * *}$ \\
\hline & Mujeres & $10,5 \pm 4,1$ & $8,7 \pm 3,4$ & $10,14 \pm 3,10$ & $9,5 \pm 4,1$ \\
\hline \multirow{2}{*}{ Sit and reach $(\mathrm{cm})$} & Varones & $-2,3 \pm 5,1$ & $-0,5 \pm 6,2$ & $-1,9 \pm 6,3$ & $-2,0 \pm 7,5$ \\
\hline & Mujeres & $0,9 \pm 6,9 * * *$ & $1,9 \pm 7,2^{* *}$ & $1,3 \pm 5,3 * * *$ & $-1,0 \pm 1,4^{* * *}$ \\
\hline \multirow{2}{*}{ Talla $(\mathrm{cm})$} & Varones & $168,0 \pm 7,4^{* * *}$ & $171,3 \pm 7,2 * * *$ & $173,6 \pm 5,5 * * *$ & $172,0 \pm 7,8^{* * *}$ \\
\hline & Mujeres & $161,5 \pm 5,3$ & $163,1 \pm 5,6$ & $165,4 \pm 5,6$ & $166,5 \pm 5,1$ \\
\hline \multirow{2}{*}{ Peso $(\mathrm{kg})$} & Varones & $66,8 \pm 12,1^{* * *}$ & $64,7 \pm 9,1 * * *$ & $67,9 \pm 10,9^{* * *}$ & $71,7 \pm 20,9^{*}$ \\
\hline & Mujeres & $56,5 \pm 7,7$ & $60,6 \pm 9,1$ & $62,3 \pm 10,9$ & $67,1 \pm 10,9$ \\
\hline \multirow{2}{*}{ Índice de Masa Corporal $\left(\mathrm{kg} / \mathrm{m}^{2}\right)$} & Varones & $23,8 \pm 4,5^{* * *}$ & $22,0 \pm 2,8$ & $22,7 \pm 3,7$ & $23,3 \pm 5,1$ \\
\hline & Mujeres & $21,6 \pm 2,5$ & $22,7 \pm 2,9^{*}$ & $22,8 \pm 3,5$ & $24,3 \pm 4,7$ \\
\hline \multirow{2}{*}{ Normopeso, $n(\%)$} & Varones & $58(32,6)$ & $113(52,8)$ & $103(51,8)$ & $96(46,8)$ \\
\hline & Mujeres & $120(67,4)^{* * * *}$ & $101(47,2)$ & $96(48,2)$ & $109(53,2)$ \\
\hline \multirow{2}{*}{ Sobrepeso, $n(\%)$} & Varones & $36(76,6)^{* * *}$ & $12(37,5)$ & $24(38,7)$ & $16(53,3)$ \\
\hline & Mujeres & $11(23,4)$ & $20(62,5)$ & $38(61,3)$ & $14(46,7)$ \\
\hline \multirow{2}{*}{ Obesidad, $n(\%)$} & Varones & $28(90,3)^{* * *}$ & $4(80,0)$ & $6(75,0)$ & $12(46,2)$ \\
\hline & Mujeres & $3(9,7)$ & $1(20,0)$ & $2(25,0)$ & $14(53,8)$ \\
\hline \multicolumn{6}{|l|}{ Coordinación } \\
\hline \multirow{2}{*}{ Carrera de obstáculos (seg.) } & Varones & $10,5 \pm 1,0^{* * *}$ & $10,0 \pm 0,8^{* * *}$ & $9,9 \pm 0,6^{* * *}$ & $10,3 \pm 0,8^{* * *}$ \\
\hline & Mujeres & $12,8 \pm 0,9$ & $12,7 \pm 1,1$ & $12,7 \pm 1,1$ & $12,9 \pm 1,1$ \\
\hline \multirow{2}{*}{ Manejo de balón (seg.) } & Varones & $11,6 \pm 1,3^{* * *}$ & $11,0 \pm 0,9^{* * *}$ & $10,9 \pm 0,9 * * *$ & $11,3 \pm 1,0^{* * * *}$ \\
\hline & Mujeres & $13,4 \pm 1,9$ & $13,2 \pm 2,2$ & $12,7 \pm 1,7$ & $13,0 \pm 1,7$ \\
\hline \multirow[b]{2}{*}{ Conducción de balón (seg.) } & Varones & $14,5 \pm 2,5 * * *$ & $14,7 \pm 2,8^{* * *}$ & $14,2 \pm 2,3 * * *$ & $15,2 \pm 2,5^{*} * *$ \\
\hline & Mujeres & $21,6 \pm 5,9$ & $21,2 \pm 7,3$ & $20,3 \pm 4,9$ & $21,0 \pm 8,6$ \\
\hline \multirow{2}{*}{ Lanzamiento-recepción (n) } & Varones & $38,2 \pm 5,2^{* * *}$ & $39,5 \pm 7,0^{* * *}$ & $40,6 \pm 4,9^{* * *}$ & $38,3 \pm 6,1^{* * *}$ \\
\hline & Mujeres & $31,4 \pm 4,6$ & $32,6 \pm 5,8$ & $33,4 \pm 4,6$ & $30,1 \pm 8,0$ \\
\hline
\end{tabular}

En la tabla 6 se muestra la relación entre el estado de peso, los test de coordinación y condición física en varones, mostrando el grupo en normopeso mejores promedios en abdominales, condición física, carrera de obstáculos y manejo de balón que sus pares homólogos con sobrepeso ( $p$ entre $<0,05$ $\mathrm{y}<0,001)$, y registros superiores en Ruffier, carrera de obstáculos, manejo de balón, conducción de balón y lanzamiento-recepción que sus semejantes con obesidad ( $p$ entre $<0,05 y<0,001$ ). En la comparación entre los grupos con exceso de peso, aquellos con sobrepeso mostraron un mejor rendimiento físico en abdominales, carrera de obstáculos, manejo de balón, conducción de balón, lanzamiento-recepción ( $p$ entre $<0,05 \mathrm{y}<0,01$ ) que sus pares homólogos con obesidad. Por último, el grupo con obesidad mostró valores más elevados en coordinación que sus semejantes con normopeso o sobrepeso ( $p<0,001$, en ambos casos).

Respecto a las mujeres, el grupo en normopeso obtuvo registros superiores en dinamometría ma-

de las variables tanto varones como mujeres alcanzan su mejor rendimiento físico en las edades inferiores, tal y como sucede a los 14 años en los varones con el test sit and reach $\mathrm{y}$ en las mujeres con el test de abdominales. nual, manejo de balón y conducción de balón $(p<0,01)$ con respecto a sus semejantes con sobrepeso; y en Ruffier, sit and reach y coordinación ( $p$ entre $0,05 \mathrm{y}<0,001)$ al compararse con el grupo de obesidad. El grupo con sobrepeso 
Tabla 5 .

alores percentiles de la batería Condición Física relacionada con la Salud (COFISA) en mujere de 14-17 años.

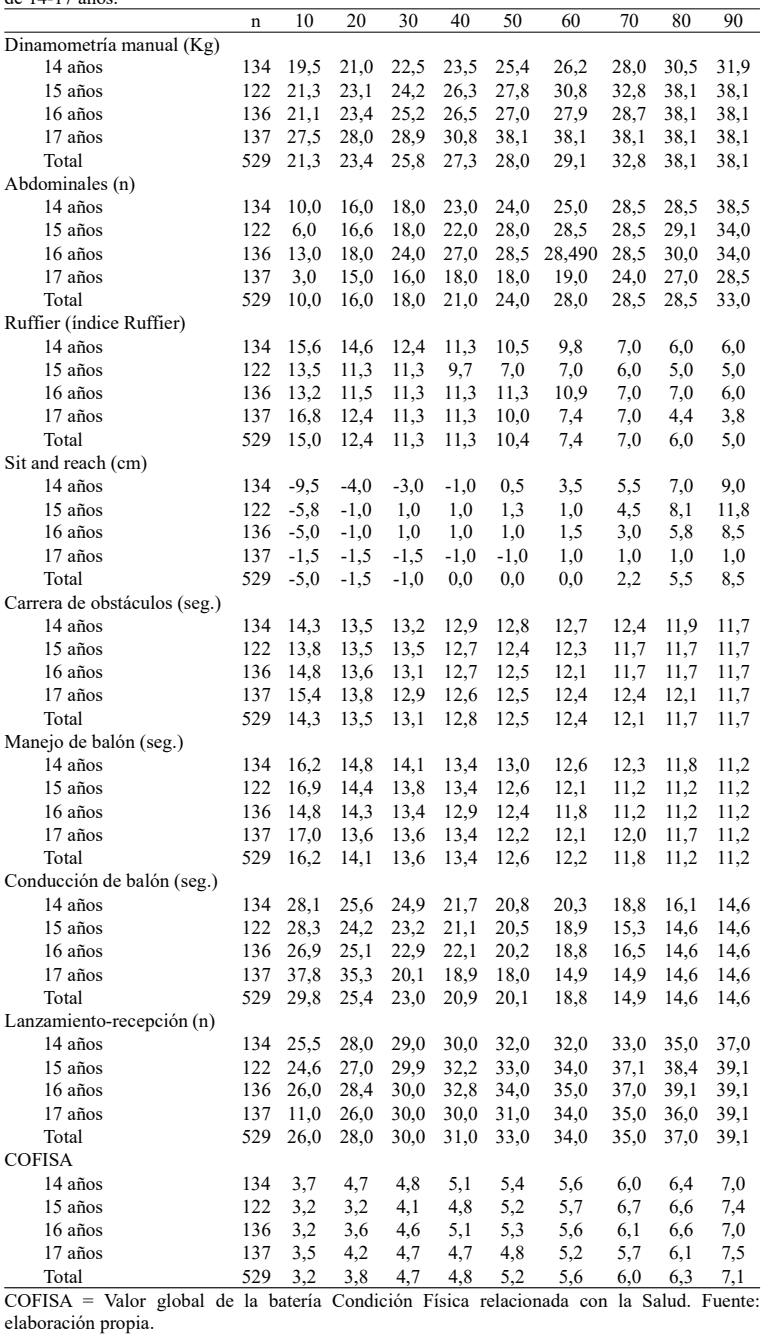

\section{Discusión}

El objetivo de este estudio fue proporcionar valores percentiles del test de valoración de las capacidades coordinativas y condicionales en adolescentes de 14 a 17 años de edad, estableciendo las diferencias según el género y el estado de peso corporal. Estos percentiles pueden ser utilizados como patrón de referencia para la valoración del desarrollo funcional o el diagnóstico clínico de enfermedades crónicas. En este sentido, los docentes de Educación Física cuentan con una herramienta válida y fiable (COFISA) para evaluar las tendencias de la condición física relacionada con la salud conforme se avanza con la edad en esta fase etaria previa a la juventud (Rosa-Guillamón, 2017; LázaroMartínez, 2011).

Respecto a los resultados obtenidos, la prueba MANOVA reflejo que los varones obtienen un mayor rendimiento físico a excepción del test de flexibilidad y el estado de peso en normopeso, situándose ambos elementos a favor de las mujeres. Resultados similares a otros estudios (Lopes et al., 2018; Palomino-Devia et al., 2016; Secchi et al., 2014) donde señalan que durante la adolescencia, la fuerza muscular se ve afectada por la maduración biológica; la mayor producción de hormonas anabólicas que ocurre durante la pubertad a favor de los varones puede afectar a la hipertrofia muscular y los niveles de fuerza muscular (Silva et al., 2012), además de la interacción de factores biológicos (crecimiento de tejido adiposo, maduración sexual) y culturales como cambios en los intereses sociales, la presión de grupo y falta de motivación pueden condicionar estas diferencias (Bustamante, Beunen, \& Maia, 2012). Sin embargo, se ha descrito que los varones poseen menor flexibilidad para la flexión/extensión de hombro, flexión / extensión de tronco, sentarse y alcanzar, elevación del hombro y muñeca, extensión del tronco y cuello (Espada, Montesinos, \& Vicente, 2007), debido a las diferencias en la morfología del esqueleto entre los dos sexos, el estrógeno circulante, un porcentaje más pronunciado de exceso adiposo, menor masa muscular y, producción de relaxina a favor de las mujeres provoca que obtengan mayores promedios de rango articular (Docherty $\&$ Bell, 1985).

A su vez, los varones obtuvieron un mayor IMC a la edad de 14 años, a los 16 años una mayor coordinación; y a los 17 años una condición física global respecto a las mujeres (véase tabla 3). Resultados similares a otros estudios (Ortega et al., 2011) donde se observa un dimorfismo sexual significativo a partir de los 12 años (Marrodán-Serrano et al., 2009), pudiendo contribuir las tendencias en las dimensiones corporales a la explicación de las tendencias seculares en fuerza y rendimiento del aparato locomotor a favor de los varones (Malina, 2004).

Por ello, se requieren estudios que tengan en cuenta el ritmo, tiempo de crecimiento y maduración psicosomática, particularmente en las mujeres (Eisenmann, Laurson, \& Welk, 2011).

En general, con el avance de la edad se observa un incremento progresivo del nivel de CF global; hallando su mayor

rendimiento a los 14 años los varones en flexibilidad y en las reach, condición física y coordinación ( $p$ entre $0,05 \mathrm{y}<0,001$ ) con respecto al grupo con normopeso; $y$ en Sit and Reach $(p$ $<0,05)$ y coordinación $(p<0,01)$ en relación al grupo con obesidad, siendo el grupo con obesidad quien mostró valores más elevados en abdominales $(p<0,002)$ (Véase tabla 7) 
mujeres en la capacidad musculo-esquelética abdominal (Véase tablas 4 y 5). Resultados similares a otros estudios (Moro et al., 2016) donde parece que el rendimiento de las pruebas de aptitud física tiende a alcanzar un máximo desde alrededor de los 15 años, en las mujeres principalmente (Tambalis et al., 2016; Piccinno, \& Colella, 2014). A su vez, estas diferencias pueden ser debidas a que los adolescentes de ambos sexos en la etapa de maduración puberal muestran valores más altos de VO2max en comparación con los adolescentes en la etapa pos puberal (Silva, \& Martins, 2017). Asimismo, estas diferencias con edades más prepúberes, puede ser debido a que con el inicio de la pubertad se produce un aumento en la sección transversal del músculo debido a la presencia de la hormona del crecimiento, lo que resulta en un aumento de la concentración y actividad enzimática, así como en la concentración del músculo y del lactato en sangre (Rowland, 1996). Este factor provoca un aumento en el rendimiento físico debido al aumento del volumen sanguíneo, el aumento del tamaño del corazón, la mayor cantidad de glóbulos rojos circulantes y una tasa de hemoglobina más pronunciada, que afecta favorablemente el transporte de oxígeno de los pulmones a otros tejidos (Soares et al., 2014). De igual modo, el género, edad, altura y peso son todos importantes predictores de rendimiento en medidas de fuerza muscular (Rosa-Guillamón, 2017)

En la relación entre el estado de peso, los test de coordinación y condición física, los varones en normopeso mostraron mayores promedios que sus pares en sobrepeso y obesidad. Cabe destacar que el grupo en obesidad mostró valores más elevados en coordinación que sus semejantes con normopeso o sobrepeso (Véase tabla 6). Resultados que difieren de algunos estudios (Cruz Estrada, Tlatempa Sotelo, Valdes-Ramos, Hernández Murúa, \& Manjarrez-Montes-de-Oca, 2017) donde señalan que el aumento progresivo de la adiposidad conforme aumenta la edad limita negativamente el desempeño de la fuerza de las extremidades inferiores debido al lastre que supone este exceso de peso (Sepúlveda-Cáceres et al., 2018) encontrándose en multitud de estudios que los escolares en normopeso versus sobrepeso/obesidad fueron significativamente mejores que sus compañeros en cuatro estructuras: equilibrio, esquema corporal, organización espacial y organización temporal (Bucco-dos Santos, \& Zubiaur-González, 2013) aunque con distinto ritmo en cada uno de los sexos (especialmente desde las edades peripuberales) (Marrodán-Serrano et al., 2009). Sin embargo, la nueva paradoja de la obesidad es que la delgadez por sí misma no es sinónimo de salud y de esperanza de vida saludable, es decir, personas catalogadas con exceso de grasa, pero en forma (fat but fit) pueden tener mayor salud respecto a sus pares en normopeso. Esta razón puede ser el motivo por el cual los escolares en obesidad posean mayores promedios en coordinación (Ortega, Ruiz, \& Castillo, 2013), además de la falta de una buena coordinación motriz está asociada a la falta de actividad física (GarcíaCantó, Pérez, Rodríguez, \& Moral, 2013).

Por su parte, las mujeres en normopeso obtuvieron registros superiores en fuerza manual y coordinación respecto al grupo en sobrepeso y obesidad, siendo el grupo en sobrepeso quien mostró registros superiores en abdominales, resistencia cardiovascular, flexibilidad, condición física y coordinación con respecto al grupo con normopeso; y en flexibilidad y coordinación en relación al grupo con obesidad, siendo el grupo con obesidad quien mostró valores más elevados en fuerza abdominal (Véase tabla 7). Resultados similares a otros estudios donde la aptitud cardiorrespiratoria se asoció con la grasa corporal alta, independientemente del nivel de maduración sexual (Minatto et al., 2016; Gualteros et al., 2015). Sin embargo, en otros estudios, el aumento del estado del peso tuvo una asociación negativa con las medidas de fuerza que implicaban el levantamiento del cuerpo, pero se asoció con un mejor desempeño en las pruebas que no implicaban el levantamiento del cuerpo (Ervin, Fryar, Wang, Miller, \& Ogden, 2014).

En este sentido, la prescripción de ejercicio físico desde el punto de vista de la salud se compone de ejercicio aeróbico y entrenamiento de fuerza, además de los ejercicios destinados a la mejora de la amplitud del movimiento (movilidad articular), mejora del equilibrio y mejora de la coordinación (Rosa-Guillamón, 2017). En personas con obesidad o sobrepeso se sugiere comenzar por un entrenamiento centrado en ejercicios de fuerza, caracterizados por una dinámica intermitente, por su mejora sobre el rendimiento motor y los beneficios sobre la reducción de lesiones (Faigenbaum, Lloyd, \& Myer, 2013). La promoción de programas de acondicionamiento neuromuscular durante la adolescencia está ampliamente recomendado, siempre y cuando estén apropiadamente supervisados y diseñados por personal cualificados (Peña, Heredia, Lloret, Martín, \& Da Silva-Grigoletto, 2016). Asimismo, duplicar la carga lectiva de Educación Física es estímulo suficiente para mejorar la CF y, concretamente, la capacidad aeróbica, componente que ha mostrado una relación muy estrecha con la salud cardiovascular en adolescentes (Ardoy et al., 2011).

\section{Limitaciones}

Lamentablemente, no se disponía de un indicador del estado de madurez biológica de la población. Esta es una limitación, ya que las referencias individuales en el tiempo y el ritmo de crecimiento en talla y peso aumentan drásticamente en poco tiempo y podrían influir en el IMC per se y, a su vez, en las relaciones entre el IMC y las medidas de ajuste en la adolescencia temprana y media. Sin embargo, estos resultados proporcionan va-liosa información del proceso de crecimiento y desarrollo en la condición física. Como fortaleza, destacamos la posible capacidad predictiva de estos percentiles para esta población escolar en la Región de Murcia. La meta a conseguir debe ser la mejora de la condición física de la población, que llevará consigo una reducción de la grasa corporal y la adopción de un estilo de vida saludable.

\section{Conclusiones}

Este estudio proporciona percentiles actualizados en una población representativa de adolescentes de la Región de Murcia. Estos percentiles permiten evaluar la condición física relacionada con salud con el fin de identificar a aquellos escolares en riesgo de enfermedades crónicas importantes así como a evaluar los efectos de intervenciones alternati- 
vas. Sobre los resultados obtenidos se concluye que I) El nivel de condición física de los varones fue superior a las mujeres a excepción de la flexibilidad y el estado de peso en normopeso. II) Con el avance de la edad se observa un incremento progresivo del nivel de condición física global; a excepción de la flexibilidad y la capacidad musculo-esquelética abdominal. III) En la relación entre el estado de peso, coordinación y condición física según el sexo, los varones en normopeso mostraron mayores promedios que sus pares en sobrepeso y obesidad, mientras que en las mujeres se hallan resultados dispares según el estado de peso.

\section{Referencias}

Ardoy, D. N., Fernández-Rodríguez, J. M., Ruiz, J. R., Chillón, P., España-Romero, V., Castillo, M. J., \& Ortega, F. B. (2011). Mejora de la condición física en adolescentes a través de un programa de intervención educativa: Estudio EDUFIT. Revista Española de Cardiología, 64(6), 484-491.

Barrancos SL (2008). Fiabilidad y validez de un protocolo de evaluación de la condición física relacionada con la salud (COFISA) en escolares (Tesis de Maestría). Universidad de Murcia. Departamento de Expresión plástica, musical y dinámica. Murcia.

Bucco-dos Santos, L., \& Zubiaur-González, M. (2013). Desarrollo de las habilidades motoras fundamentales en función del sexo y del índice de masa corporal en escolares. Cuadernos de psicología del Deporte, 13(2), 63-72.

Bustamante, A., Beunen, G., \& Maia, J. (2012). Valoración de la aptitud física en niños y adolescentes: construcción de cartas percentílicas para la región central del Perú. Revista Peruana de Medicina Experimental y Salud Pública, 29, 188-197.

Casajús, J. A., Leiva, M. T., Ferrando, J. A., Moreno, L., Aragonés, M. T., \& Ara, I. (2006). Relación entre la condición física cardiovascular y la distribución de grasa en niños y adolescentes. Apunts. Medicina de l'esport, 41(149), 7-14.

Castro-Piñero, J., Ortega, F.B., Keating, X.B., GonzálezMontesinos, J.L., Sjöstrom, M. \& Ruiz, J.R. (2011). Percentile values for aerobic performance running/ walking field tests. in children aged 6 to 17 years; influence of weight status. Nutr Hosp, 26(3), 572-578

Cole, T. J., Bellizzi, M. C., Flegal, K. M. y Dietz, W. H. (2000). Establishing a standard definition for child overweight and obesity worldwide: international survey. British Medical Journal, 320, 1240-1243. doi:10.1136/bmj.320. 7244.1240.

Cruz Estrada, F. D. M., Tlatempa Sotelo, P., Valdes-Ramos, R., Hernández Murúa, J. A., \& Manjarrez-Montes-de-Oca, R. (2017). Overweight or Obesity, Gender, and Age Influence on High School Students of the City of Toluca's Physical Fitness. BioMed research international, 2017.

Docherty D, Bell R. D., (1985). The relationship between flexibility and linearity measures in boys and girls 6-15 years of age. Journal of human movement studies (Edinburgh, Eng.) 11(5), 279-288.

Eisenmann, J. C., Laurson, K. R., \& Welk, G. J. (2011). Aerobic fitness percentiles for U.S. adolescents. American Journal of Preventive Medicine, 41, S106-S110. doi:10.1016/ j.amepre.2011.07.005

Ervin, R. B., Fryar, C. D., Wang, C. Y., Miller, I. M., \& Ogden, C. L. (2014). Strength and body weight in US children and adolescents. Pediatrics, peds-2014.

Espada, D. R., Montesinos, J. L. G., \& Vicente, J. M. (2007). Diferencias en las amplitudes articulares entre varones y mujeres en edad escolar. Apunts. Medicina de l'esport, 42(153), 13-25.

Faigenbaum, A. D., Lloyd, R. S., \& Myer, G. D. (2013). Youth resistance training: past practices, new perspectives, and future directions. Pediatric exercise science, 25(4), 591604.

García-Cantó, E., Pérez-Soto, J. J., Rodríguez, P. L., \& Moral, J. E. (2013). Nivel de actividad física y su relación con la condición física saludable en adolescentes. Trances, 5(3), 213-228.

Gualteros, J. A., Torres, J. A., Umbarila-Espinosa, L. M., Rodríguez-Valero, F. J., \& Ramírez-Vélez, R. (2015). Una menor condición física aeróbica se asocia con alteraciones del estado de salud en niños y adolescentes de Bogotá, Colombia. Endocrinología y Nutrición, 62(9), 437446.

Guedes, D. P., Franzini, P. C., Júnior, R. P., \& Morales, J. M. M. (2017). Antropometria e Aptidão Física de Adolescentes Latino-Americanos Anthropometry and Physical Fitness of Latin American Adolescents. Retos, 31, 264-270.

Lázaro-Martínez, C. J. (2011). Hábito de consumo de alcohol y su relación con la condición física saludable en adolescentes de la Región de Murcia. [Tesis Doctoral]. Murcia: Universidad de Murcia.

Lopes, V. P., Malina, R. M., Gomez-Campos, R., CossioBolaños, M., de Arruda, M., \& Hobold, E. (2018). Body mass index and physical fitness in Brazilian adolescents. Jornal de pediatria.

Malina RM. 2004. Secular trends in growth, maturation and physical performance: a review. Anthropol Rev 67:3-31.

Marrodán-Serrano, M., Collazos, J. R., Romero, S. M., Santurino, M. M., Armesilla, M. C., del Cerro, J. P., \& de Espinosa, M. G. M. (2009). Dinamometría en niños y jóvenes de entre 6 y 18 años: valores de referencia, asociación con tamaño y composición corporal. In Anales de pediatría (Vol. 70, No. 4, pp. 340-348). Elsevier Doyma.

Minatto, G., de Sousa, T. F., de Carvalho, W. R. G., Ribeiro, R. R., Santos, K. D., \& Petroski, E. L. (2016). Relação entre aptidão cardiorrespiratória e adiposidade corporal em meninas. Revista Paulista de Pediatria, 34(4), 469-475.

Moro, P. B., Castillo, M. B., Espinosa, M. G. M., Algaba, E. V., López-Ejeda, N., \& Serrano, M. D. M. (2016). Semilongitudinal analysis of physical status in Madrilenian adolescents. Archivos de Medicina del Deporte, 33(3), 183-192.

Mundín, E. O., Lafita, J., Petrina, E., \& Argüelles, I. (2009). Composición corporal y obesidad. In Anales del Sistema Sanitario de Navarra (Vol. 25, pp. 91-102).

Ortega FB, Ruiz JR, Castillo MJ, Sjöström M. (2008). Physical fitness in childhood and adolescence: a powerful marker of health.Int J Obes (Lond), 32(1), 1-11.

Ortega, F. B., Artero, E. G., Ruiz, J. R., España-Romero, V., 
Jiménez-Pavón, D., Vicente-Rodríguez, G., \& Ciarapica, D. (2011). Physical fitness levels among European adolescents: the HELENA study. British journal of sports medicine, 45(1), 20-29.

Ortega, F. B., Ruiz, J. R., \& Castillo, M. J. (2013). Actividad física, condición física y sobrepeso en niños y adolescentes: evidencia procedente de estudios epidemiológicos. Endocrinología y Nutrición, 60(8), 458-469.

Palomino-Devia, C., Otero-Saborido, F. M., \& González-Jurado, J. A. (2016). Análisis de la adiposidad y la condición física en escolares colombianos. Biomédica, 36(3), 343353.

Peña, G., Heredia, J. R., Lloret, C., Martín, M., \& Da SilvaGrigoletto, M. E. (2016). Iniciación al entrenamiento de fuerza en edades tempranas: revisión. Revista Andaluza de medicina del deporte, 9(1), 41-49.

Piccinno, A., \& Colella, D. (2014). Physical fitness level in Italian high-school adolescents: a cross-sectional study. Journal of Physical Education and Sport, 14(3), 431.

Rosa-Guillamón, A, García-Cantó, E., \& Carrillo-López, P. J. (2018). La educación física como programa de desarrollo físico y motor. EmásF: revista digital de educación física, (52), 105-124.

Rosa-Guillamón, A. (2017). Análisis bibliográfico de las baterías de evaluación de la condición física. Rev.peru.cienc.act.fis.deporte, 4(4), 533-543.

Rowland, T.W., 1996. Developmental Exercise Physiology. Human Kinetics, Champaign.

Ruiz J. R., Castro-Piñero J., Artero E. G., Ortega F.B., et al. (2009). Predictive validity of health-related fitness in youth: a systematic review. Br J Sports Med, 43(12), 90923.

Secchi, J. D., García, G. C., España-Romero, V., \& Castro-Piñero,
J. (2014). Condición física y riesgo cardiovascular futuro en niños y adolescentes argentinos: una introducción de la batería ALPHA. Archivos argentinos de pediatría, 112(2), 132-140.

Sepúlveda-Cáceres, Méndez Cornejo, J., Duarte Farfán, C., Herrera, M., Gómez-Campos, R., Lazari, E., \& CossioBolaños, M. (2018). Relación entre adiposidad corporal y salto horizontal en niños y adolescentes escolares. Revista chilena de pediatría (AHEAD), 89(6), 701-708.

Silva G, Aires L, Mota J, Oliveira J, et al. (2012). Normative and criterion-related standards for shuttle run performance in youth. Pediatr Exerc Sci, 24(2), 157-69.

Silva, D. A, \& Martins, P. C. (2017). Impact of physical growth, body adiposity and lifestyle on muscular strength and cardiorespiratory fitness of adolescents. Journal of bodywork and movement therapies, 21(4), 896-901.

Soares, N.M.M., Silva, R.J.S., Melo, E.V., Oliveira,A.C.C., 2014. Influence of sexual maturation on cardiorespiratory fitness in school children. Rev. Bras. Cineantropom. Desempenho. Hum. 16, 223e232.

Tambalis, K. D., Panagiotakos, D. B., Psarra, G., Daskalakis, S., Kavouras, S. A., Geladas, N., \& Sidossis, L. S. (2016). Physical fitness normative values for 6-18-year-old Greek boys and girls, using the empirical distribution and the lambda, mu, and sigma statistical method. European journal of sport science, 16(6), 736-746.

Yeste, D., \& Carrascosa, A. (2011). Complicaciones metabólicas de la obesidad infantil. In Anales de Pediatría (Vol. 75, No. 2, pp. 135-e1). Elsevier Doyma.

Yuste J.L., Rodríguez P.L. (2012). Fiabilidad intra e interexplorador y validez de pruebas de evaluación condicionales en escolares. ii congreso de la Asociación española de ciencias del Deporte; Madrid, España. Madrid: InEF.

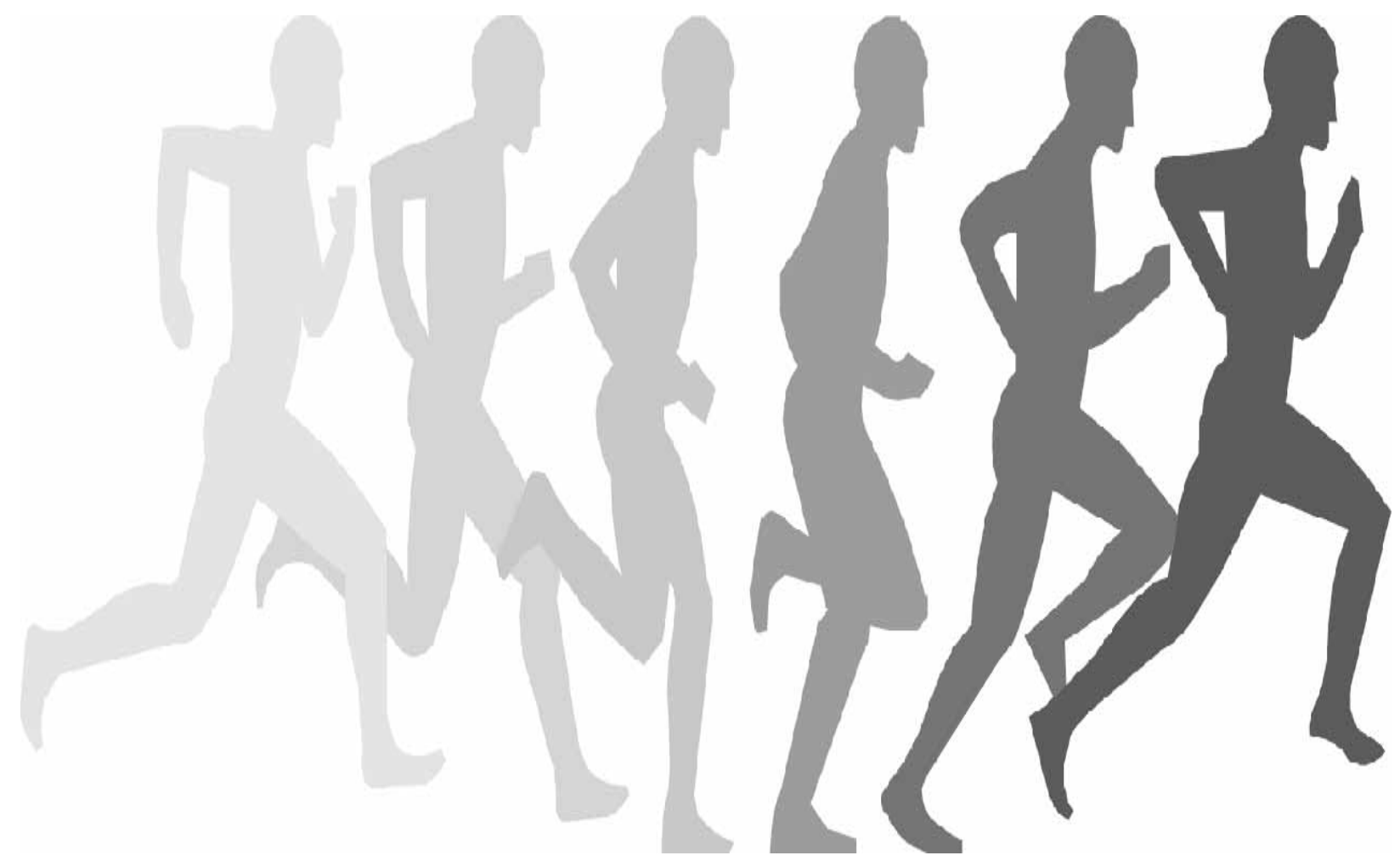

